\title{
Los Riesgos para Mujeres Asociados a la Dispensación de Cyclofem en Droguerías de Bogotá D.C. Revisión de Alcance
}

\author{
*Germán Clavijo Cohén ${ }^{1}$ \\ ${ }^{1}$ Servicio Nacional de Aprendizaje (SENA)-Sistema de Investigación Desarrollo Tecnológico e Innovación \\ (SENNOVA)-Centro de formación Talento Humano en Salud (CFTHS), Regional Distrito Capital, Colombia
}

Cómo referenciar este artículo/ How to reference this article:
Clavijo Cohén G. Los Riesgos para Mujeres Asociados a la Dispensación de Cyclofem en Droguerías de Bogotá D.C. Revisión de Alcance.Mem. Inst. Investig. Cienc. Salud. $2018 ; 16(2)$ : 100-106

\section{R E S U M E N}

La normatividad legal en Colombia exige que la dispensación y administración de Cyclofem en droguerías esté apoyada por una prescripción médica. Cyclofem es un anticonceptivo inyectable de combinación de vía intramuscular (AIC) que contiene medroxiprogesterona acetato y cipionato de estradiol y presenta un $99,7 \%$ de efectividad. Este medicamento produce sangrados abundantes, prolongados e irregulares, náuseas, mareos, aumento de peso, dolor de cabeza, osteoporosis y aumenta la tolerancia a la glucosa y la resistencia a la insulina. En la ciudad de Bogotá, D.C. sólo el 0,4\% de las droguerías exigen la prescripción médica para la dispensación y administración de dicho medicamento.

Palabras clave: droguerías, Cyclofem, prescripción médica, Dispensación, administración intramuscular.

\section{The Risks for Women Associated with the Dispensation of Cyclofem in Pharmacies of Bogotá D.C. A Scope Review}

\author{
A B S T R A C T
}

The legal regulations in Colombia require that the dispensing and administration of Cyclofem in drugstores be supported by a medical prescription. Cyclofem is an injectable intramuscular combination contraceptive (CCI) that contains medroxyprogesterone acetate and estradiol cypionate with an effectiveness of $99.7 \%$. This medication causes heavy, prolonged and irregular bleeding, queasiness, dizziness, weight gain, headache, osteoporosis and increases glucose tolerance and insulin resistance. Only $0.4 \%$ of the pharmacies in Bogotá D.C. demanded the presentation of the medical prescription for dispensing and intramuscular administration (I.M) of Cyclofem in accordance with the current legal regulations.

Keywords: drugstores, Cyclofem, medical prescription, dispensation, intramuscular administration.

\section{DESARROLLO DEL TEMA}

El Ministerio de Salud y Protección Social (MINSALUD) ${ }^{(1)}$ y El Concejo Nacional de Política Económica y Social ${ }^{(2)}$ establecen que los servicios farmacéuticos de baja complejidad ambulatorios, también conocidos como droguerías tienen como objetivos: 1. promover y propiciar estilos de vida saludables, 2. prevenir factores de riesgo derivados del uso inadecuado de medicamentos y dispositivos médicos y promover su uso adecuado y 3. suministrar los medicamentos y dispositivos médicos e informar a los pacientes sobre su uso adecuado. Frente lo anterior, MINSALUD ${ }^{(3,4,5)}$ determinó que los medicamentos 
inyectables para su comercialización y administración por vía intramuscular DEBEN de forma obligatoria incluir en sus etiquetas y empaques la leyenda "VENTA BAJO FÓRMULA MÉDICA" y que las droguerías DEBEN contar con un sistema de gestión de calidad que garantice los principios de oportunidad, seguridad, eficiencia y calidad, junto con lo formación académica y entrenamiento de su personal.

La Organización Mundial de la Salud $(\mathrm{OMS})^{(6)}$ define que la elección anticonceptiva depende de la eficacia del método anticonceptivo en la prevención de un embarazo no planificado y de cuán correcta y consistentemente se le usa. Agrega, que el anticonceptivo inyectable combinado (AIC) (Lunelle $\AA /$ Acetato de medroxiprogesterona y Cipionato de estradiol) presenta un $99,7 \%$ de eficiencia durante su primer año de uso, pero que su administración debe estar ajustada a los criterios de riesgos para cada mujer en edad fértil y que sólo deben ser determinados por el médico tratante. Lo anterior se acompasa con la adopción por parte de MINSALUD de la norma técnica para la atención de planificación familiar, la cual enfatiza que los beneficios anticonceptivos están asociados a la forma correcta y consistente de los $\operatorname{AIC}^{(7)}$.

El estado colombiano define que la fecundidad es la capacidad reproductiva de una población, particularmente entre los 15 a 49 años; dicha capacidad actualmente alcanza los 74 nacimientos por 100.000 habitantes, lo que se contrapone con la tasa de mortalidad materna que se encuentra en 71,6 muertes por cada 100.000 nacimientos vivos ${ }^{(8,9)}$. Otras estadísticas gubernamentales revelan que para el año 1967 el promedio de hijos por mujer $(\mathrm{h} / \mathrm{m})$ fue de 6,7 , mientras que para el año 2015 fue de $2 \mathrm{~h} / \mathrm{m}$, pero las zonas rurales del país presentan una tasa de $2,6 \mathrm{~h} / \mathrm{m}$, siendo particularmente visible esta última situación en Región Atlántica con 2,5 h/m. Es importante enfatizar que los estratos socioeconómicos bajos tienen prácticamente el doble de hijos que los estratos socioeconómicos altos ${ }^{(9)}$. Si a todo lo antes expuesto, se le suma que para el 2015 la edad promedio para el primer embarazo se encontraba entre los 15 a 19 años, que cerca del 32,6\% de los métodos AIC son suministrados por las droguerías y que los métodos AIC son el segundo método anticonceptivo más utilizado por las mujeres entre 15 a 19 años ${ }^{(10,11)}$, se hace necesario evaluar el cumplimiento de las políticas públicas en materia de seguridad al paciente en lo concerniente a la dispensación y administración en las droguerías de acetato de medroxiprogesterona/cipionato de estradiol (Cyclofem).

El Cyclofem es un anticonceptivo combinado inyectable de uso mensual recomendado por la OMS para reducir la natalidad no deseada debido a su seguridad y efectividad, comoquiera que su tasa de efectividad es del 99,7\% siempre y cuando su uso se ajuste a lo prescripto por el facultativo. Estadísticas muestran que las mujeres en edad fértil en Iraq $(12,6 \%)$, Indonesia $(66,5 \%)$, Turquía $(28,2 \%)$ y México $(25,1 \%)$ eligen al Cyclofem como su método anticonceptivo. No obstante a lo anterior, las mujeres que usan Cyclofem durante el primer año reportan amenorrea (14,7\%), sangrado prolongado $(15,8 \%)$, sangrado irregular $(9,4 \%)$, sangrado frecuente $(7,6 \%)$, náuseas $(18 \%)$, dolor de cabeza $(6,1 \%)$, aumento de peso $(2,2 \%)$ y mareo $(1,8 \%)^{(12-14)}$; los efectos secundarios anteriormente descritos están asociados al acetato de medroxiprogesterona (MPA) ${ }^{(15)}$. En relación con el aumento de peso, se define que el uso de MPA está relacionado con el origen geográfico de la mujer, toda vez que las mujeres de origen europeo y asiático tienen casi el doble de probabilidad de aumentar de peso que las mujeres de origen americano ${ }^{(16)}$.

Ahora bien, la osteoporosis afecta a más de 200 millones de mujeres en el mundo y ocasiona cerca de 8,9 millones de fracturas anualmente y el uso prologando de corticoides aumenta en un factor de 2 el riesgo de fracturas ${ }^{(17)}$. El uso de MPA reduce la densidad mineral de los huesos, particularmente en mujeres menores de 20 años y mayores de 50 años, respectivamente, aumentando el riesgo de fracturas, por esta razón la agencia Food and Drug Administration (FDA) estableció de forma obligatoria que todos los productos que contengan MPA advirtieran a la usuaria sobre el riesgo de desmineralización ósea ${ }^{(18,19)}$. No obstante, la OMS considera al MPA de bajo riesgo para las mujeres entre 18 a 45 años (Categoría I) y para mujeres de mayor edad lo considera de riesgo moderado (Categoría 2). Ahora bien, la OMS advierte que en el caso de mujeres con diabetes que presenten cuadros clínicos con evidente daño vascular no se debe administrar MPA bajo ninguna circunstancia; estudios demuestran que el MPA cambia el metabolismo de la glucosa, ya que disminuye la tolerancia a la glucosa y aumenta la resistencia a la insulina ${ }^{(20)}$.

Es necesario resaltar que el Cyclofem produce más efectos secundarios que los métodos anticonceptivos hormonales de tipo oral (MAHO), ya que este presenta mayor incidencia de 
manchado vaginal, sensibilidad en los senos, acné y ganancia de peso al cabo de seis (6) meses de uso comparado con los $\mathrm{MAHO}^{(21)}$.

Con el fin de controlar la natalidad el Plan Obligatorio de Salud (POS) en Colombia contempla que las mujeres durante su embarazo reciban atención médica en lo referente a su salud sexual y reproductiva y es por esto, que dentro de esta atención se administran a la mujeres post-parto un AIC; enlazado con lo anterior, se demostró que las bebés de mujeres que recibieron por 130 días MPA no presentaron residuos o metabolitos secundarios de MPA en su orina ni alteraciones de la hormona folículo estimulante (FSH) y la hormona luteinizante $(\mathrm{LH})^{(22)}$; por lo otro lado, no es contundente la presencia de MPA en la leche materna, pero si se señala que este tipo de anticonceptivos favorecen la ganancia de peso en los bebés(23).

Luego de establecer los efectos secundarios del Cyclofem, no se puede dejar de lado un fenómeno social que está afectando a las mujeres que utilizan AIC, el cual consiste en una relación entre el uso de AIC y el incremento del riesgo de adquirir una enfermedad de transmisión sexual como el SIDA, herpes simple tipo 2, gonorrea, tricomoniasis y clamidiasis $^{(24)}$.

En cuanto al ámbito nacional, el método de planificación hormonal es el método más utilizado por las mujeres con un $60 \%$, en donde el $98 \%$ de las mujeres que planifican con cualquier método de anticoncepción lo usan adecuadamente y el $90 \%$ de ellas tiene la autonomía de elegir el método anticonceptivo ${ }^{(25)}$.

\section{Panorama de la ética a nivel mundial en el sector farmacéutico y riesgos a la salud de la mujer en edad fértil}

A nivel mundial se han identificado cinco fuentes de corrupción en el sector farmacéutico, 1. pocas campañas de concientización encaminadas a reducir la corrupción en la comunidad, 2. sistemas de investigación y justicia deficientes, 3. ausencia de agencias anticorrupción, 4. poca transparencia y responsabilidad en los procesos de adquisición y 5 . leyes inexistentes o flexibles frente al monopolio o los incentivos por ventas o formulación. Todo lo anterior ocasiona que los servicios en salud asociados a medicamentos se conviertan en un agente de inequidad, inseguridad y sobrecosto. Por lo anterior, Estados Unidos de América (EUA) en el año 2002 restringió y Alemania en el año 2008 prohibió que los médicos recibieran cualquier tipo de beneficio por parte de las industrias farmacéuticas, con el único propósito de evitar influencias sobre sus hábitos de prescripción. En el ámbito nacional la Ley 23 de 1981 (Código de ética médica) en su artículo 40 expresamente prohíbe al cuerpo médico recibir dádivas o beneficios de farmacias y laboratorios ${ }^{(26)}$. EUA entre los años 1993-2001 y en la India entre los años 2001-2005 conformaron agencias anticorrupción cuyo propósito era detectar posibles focos de corrupción dentro del sistema de salud. Bajo esta orientación Kirguistán en el año 2001 estableció políticas de transparencia y responsabilidad en el sector ${ }^{(27)}$.

En virtud de lo anterior, el papel de los profesionales en los servicios farmacéuticos migra de simplemente entregar un medicamento a un paciente de forma distante e indiferente a un proceso controlado de dispensación, por consiguiente dichos profesionales deben orientar sus prácticas hacia el uso racional del medicamento y promover hábitos saludables, en otras palabras, los profesionales en los servicios farmacéuticos deben brindar la mayor y mejor información al paciente sobre el uso de los medicamentos y sus efectos secundarios, más cuando de forma concomitante o secuencial se administran varios de estos ${ }^{(2,28)}$.

La OMS insiste que los profesionales de los servicios farmacéuticos deben cumplir con siete $^{(7)}$ lineamientos (The Seven-star pharmacist): 1. Excelencia en la comunicación, 2. Excelencia en la gerencia de los procesos, 3. Excelencia en la toma de decisiones, 4. Excelencia en el cuidado al paciente, 5. Excelencia en el liderazgo, 6. Excelencia en la enseñanza y 7. Excelencia en el autoaprendizaje ${ }^{(29)}$.

Dentro del sector farmacéutico se enfatiza que los profesionales de los servicios farmacéuticos deben mejorar la calidad de vida de sus usuarios, esto implica a) identificar los potenciales problemas relacionados con los medicamentos, b) resolver los actuales problemas relacionados con los medicamentos y c) prevenir los potenciales problemas relacionados con los medicamentos. Estos tres conceptos más los siete lineamientos de la OMS derivan en la gerencia de la terapia de medicación (MTM por sus siglas en inglés). La mencionada gerencia se estructura a partir de una revisión constante del régimen de medicación, el registro juicioso de la medicación y un plan de acción relacionado con 
medicamentos, el cual incluye recomendaciones terapéuticas, remisiones, historiales y seguimientos, por eso, los servicios farmacéuticos deben articularse con los demás profesionales de la salud, para que en conjunto se logre un tratamiento integral a las patologías ${ }^{(28)}$.

Resultado de todo lo anterior, la OMS está promoviendo las buenas practicas de la farmacia (GPP por sus siglas en inglés) y es notorio como los países desarrollados marcan diferencias en su implementación comparados con los países en vía de desarrollo. Estas buenas prácticas de la farmacia alivian los sistemas de salud, reducen costos, aumentan la efectividad terapéutica, promueven estilos de vida saludable y reducen la automedicación y la inequidad ${ }^{(28)}$.

Resultados desalentadores demuestran que sólo el 0,4\% de las Droguerías de Bogotá D.C. exigen de acuerdo con la normatividad legal vigente la prescripción médica para la dispensación y administración intramuscular (I.M) de Cyclofem ${ }^{(30)}$.

Hoy en día, la industria farmacéutica colombiana presenta formas desorientadas de presión encaminadas al aumento de sus réditos, como las dádivas a los médicos, comisiones de venta a los droguistas, asociaciones de usuarios patrocinadas por laboratorios farmacéuticos, tratados de libre comercio privativos, "lobby" en las ramas del poder público, impulso de actos legislativos proteccionistas y flujo de capital humano entre la industria y el sector público. Es así como, a manera de ejemplos, en el año 2015 se impulsó el proyecto de Ley 246/2016c de 2016 en la Cámara de Representante del Congreso de la República de Colombia para que dentro del sistema de salud sólo se proveyeran medicamentos de marca ${ }^{(31)}$ y la Asociación de Laboratorios Farmacéuticos de Investigación y Desarrollos (AFIDRO) donó cerca de 1.859 millones de pesos a diferentes asociaciones de pacientes ${ }^{(32)}$.

La Corte Constitucional mediante sentencia C-084/2013 declaro exequible el artículo 133 de la Ley 1474 de 2011 (Estatuto Anticorrupción), el cual establece "Prohibición de prebendas o dádivas a trabajadores en el sector de la salud. Queda expresamente prohibida la promoción u otorgamiento de cualquier tipo de prebendas, dádivas a trabajadores de las entidades del Sistema General de Seguridad Social en Salud y trabajadores independientes, sean estas en dinero o en especie, por parte de las Entidades Promotoras de Salud, Instituciones Prestadoras de Salud, empresas farmacéuticas productoras, distribuidoras, comercializadoras $u$ otros, de medicamentos, insumos, dispositivos y equipos, que no esté vinculado al cumplimiento de una relación laboral contractual o laboral formalmente establecida entre la institución y el trabajador de las entidades del Sistema General de Seguridad Social en Salud"(33). La Cámara Farmacéutica de la Asociación Nacional de Industriales (ANDI) recalca en su código de ética sección 4.2 que "(...) No se deben realizar $u$ ofrecer a los profesionales de la salud regalos u obsequios para su beneficio personal, tales como entradas a espectáculos o eventos deportivos, artículos electrónicos, ni obsequios en efectivo o su equivalente, como bonos y/o tarjetas de regalos, entre otros" y agrega que "(...) No se deben realizar manifestaciones de felicitación dirigida a profesionales de la salud de forma individual que tengan valor comercial, ni efectuar obsequios personales ni patrocinar actividades sociales y/o de entretenimiento, relacionada con la celebración correspondiente ${ }^{\prime \prime(34)}$ y por su parte, el artículo 40 de código de ética médica establece que: "Está prohibido al médico en ejercicio recibir beneficios comerciales de farmacias, laboratorios, ópticas, establecimientos ortopédicos y demás organizaciones o instituciones similares encargadas del suministro de elementos susceptibles de prescripción médica"(24).

Usma ${ }^{(35)}$ lanza al sector el siguiente cuestionamiento "¿Cómo cumplir con el código de ética del sector farmacéutico, sin perder la posición en el segmento de mercado?, cuando todos los laboratorios utilizan la entrega de bonificaciones y obsequios a los médicos y droguistas detallistas que son quienes tienen el contacto y la confianza del consumidor final, hasta la fecha no se evidencia respuesta.

\section{CONCLUSIÓN}

Luego de lo anterior, es manifestó el riesgo al que están expuesto las mujeres en edad fértil que usan Cyclofem, no sólo por los efectos adversos ya descritos sino principalmente por los intereses económicos de la industria farmacéutica, que no anticipan la condición clínica integral de la mujer y su relación con los ingredientes activos del Cyclofem. Se llama la atención a las entidades de control y vigilancia estatal para que extremen los seguimientos a los droguistas, a las agremiaciones médicas e industriales para que 
fortalezcan el cumplimiento de sus códigos de ética, a los centros educativos para que dinamicen en sus estudiantes el componente de la responsabilidad social y a los profesionales del sector para que generen la cultura de la autorregulación.

\section{FINANCIAMIENTO}

Este proyecto fue desarrollado con recursos SENA-CFTHS, Regional Distrito Capital.

\section{AGRADECIMIENTOS}

El autor agradece al Dr. Gerardo Arturo Medina Rosas (Subdirector Centro de Formación Talento Humano en Salud) por su invaluable apoyo, sin el cual esta investigación no hubiera sido posible. A SENNOVA y al grupo de investigación GISS por su permanente acompañamiento.

\section{DESCARGO DE RESPONSABILIDAD}

El autor ha participado en la generación de la concepción, confección, redacción, revisión crítica y aprobación final del manuscrito y asume la responsabilidad de todas las ideas y concepciones que aparecen es este artículo. Declara que la investigación fue financiada con recursos SENNOVA-SENA y que no tiene conflicto de interés.

\section{REFERENCIAS}

1. Decreto 780 de 06 de mayo de 2016, Ministerio de Salud y Protección Social, por el cual se expide el decreto único reglamentario del sector salud y protección social. Recuperado a partir de: https://www.minsalud.gov.co/Normatividad_ Nuevo/

Decreto\%200780\%20de\%202016.pdf.

2. CONPES 155 de 30 de agosto de 2012, Concejo Nacional de política Económica y Social, por la cual se establece la Política Farmacéutica Nacional. Recuperado a partir de:

https://www.minsalud.gov.co/Documentos\% $20 y \% 20$ Publicaciones/Politica\%

20Farmac\%C3\%A9utica\%20Nacional.pdf

3. Decreto 677 de 26 de abril de 1995, Ministerio de Salud y Protección Social, por el cual se reglamenta parcialmente el Régimen de registros y Licencias de medicamentos y se dictan otras disposiciones. Recuperado a partir de: https://www.invima.gov.co/images/pdf/medi camentos/decretos/decreto 677 1995.pdf.

4. Decreto 2330 de 12 de julio de 2006, Ministerio de Salud y Protección Social, por el cual se modifica el decreto 2200 de 2005 y se dictan otras disposiciones. Recuperado a partir de: https://www.invima.gov.co/decretos-enmedicamentos/decreto-2330de-2006pdf/detail.html.

5. Resolución 1403 de 14 de mayo de 2007, Ministerio de Salud y Protección Social, por el cual se adopta el manual de calidad del servicio farmacéutico. Recuperado a partir de:

https://www.invima.gov.co/images/pdf/tecn ovigilancia/ buenas_practicas/ normatividad/Resolucion1403de2007.pdf.

6. Organización Mundial de la Salud. Criterios médicos de elegibilidad para el uso de anticonceptivos. Luxemburgo; 2012. 142p.
7. Resolución 769 de 03 de marzo de 2008, Ministerio de Salud y Protección Social, por el cual se adopta la norma técnica para la atención en planificación familiar. Recuperado a partir de: https://docs.supersalud.gov.co/PortalWeb/] uridica/OtraNormativa/R_MPS_0769_2008.p df.

8. Gómez A, Caicedo C. Plan Decenal de Salud Pública 2012-2021, Resumen Ejecutivo. Monitor estratégico. 2014;6: 64-74.

9. Departamento Administrativo Nacional de Estadística (DANE). Resumen Ejecutivo Encuesta Nacional de Demografía y Salud [Internet]. Bogotá, D.C. DANE; 2015. [Citado 27 de abril de 2018]. 96 p. Recuperado a partir de: http://profamilia.org.co/docs/Libro\%20RESU MEN\%20EJECUTIVO.pdf.

10. Gómez-Mercado C, Montoya-Vélez L. Factores sociales, demográficos, familiares y económicos relacionados con el embarazo en adolescentes, área urbana, Briceño, 2012. Rev Salud Pública. 2014; 16(3): 394-406.

11. Gómez-Camargo D, Canchila-Barrios C, Salguedo-Madrid G, Ochoa-Diaz M, RamosClason E, Malambo-García D. Salud Sexual y reproductiva en estudiantes universitarios de una institución de educación superior en Colombia. Rev Salud Pública. 2014: 16 (5); 660-672. DOI: http://dx.doi.org/10.15446/rsap.v16n5.39 998.

12. Yazdanpanah $M$, Mohammad E, Parnian A, Mohammad E, Najmeh J, Nouzar N. Acceptability and Side effects of Cyclofem once-a-month injectable contraceptive in Kerman, Iran. Iran J Reprod Med. 2010; 8 (4):191-196.

13. Tehrani F, Hashemi Z, Peykari N, Djalalinia, Sh. Effect of a monthly injectable contraceptive(Cyclofem) on mestrual pattern and lipoprotein profil. Eur 
J Contracept Reprod Health Care. 2004:9; 140.

14. Gallo MF, Grimes DA, Lopez LM, Schulf KF, D`arcangues, C. Combination injectable contraceptives for contraception (Review). Cochrane library (ON LINE).2008:4;1-82. DOI: $10.1002 / 14651858 . C D 004568 . p u b 3$.

15. Halpern V, Lopez LM, Grimes DA, Stockton LL, Gallo MF. Strategies to improve adherence and acceptability of hormonal methods of contraception (Review). Cochrane library (ON LINE). 2013:10;1-47. DOI: $10.1002 / 14651858 . C D 004317 . p u b 4$.

16. Lopez LM, Bernholc A, Chen M, Grey TW, Otterness C, Westhoff $C$, et al. Hormonal contraceptives for contraception in overweight or obese women (Review). Cochrane library (ON LINE). 2016: 8; 195. DOI: 10.1002/14651858.CD008452.pub4.

17. International Osteoporis Foundation Data \& Publications. FACTS AND STATISTICS. 2018. Recuperado a partir de: https://www.iofbonehealth.org/factsstatistics.

18. Lopez LM, Grimes DA, Schulz KF, Curtis KM, Chen, M. Steroidal contraceptives: effect on bone fractures in women (Review). Cochrane library (ON LINE). 2014:6; 1-93. DOI: 10.1002/14651858.CD006033.pub5.

19. Lopez LM, Chen M, Mullins Long S, Curtis KM, Helmerhorst FM. Steroidal contraceptives and bone fractures in women: evidence from observational studies. Cochrane library (ON LINE). 2015:7;1-59.

10.1002/14651858.CD009849.pub3.

20. Lopez LM, Grimes DA, Schulz, KF. Steroidal contraceptives: effect on carbohydrate metabolismin women without diabetes mellitus (Review). Cochrane library (ON LINE). 2014: 4;1-57. DOI: 10.1002/14651858.CD006133.pub5.

21. Bita J, Azade K, Mozhgan F, Fatemeh K, Fatemeh T. Comparing the satisfaction and efficacy of Cyclofem and contraceptive pills among females in Northern Iran: A randomized controlled trial study. J Adv Pharm Technol Res. 2014:5(4); 152-157. DOI: $10.4103 / 2231-4040.143025$.

22. Virutamasen $P$, Leepipatpaiboon $S$, Kriengsinyot $\mathrm{R}$, Vichaidith, $\mathrm{P}$, Ndavi Muia $\mathrm{P}$, Sekadde-Kigondu CB, et al. Pharmacodynamic Effects of DepotMedroxyprogesterone Acetate (DMPA) Administered to Lactating Women on Their Male Infants. Contraception 1996:54;153157.

23. Lopez LM, Grey TW, Stuebe AM, Chen M, Truitt ST, Gallo, MF. Combined hormonal versus nonhormonal versus progestin-only contraception in lactation (Review). Cochrane library (ON LINE). 2015:3;1-59. DOI: $10.1002 / 14651858 . C D 003988 . p u b 2$.
24. Guttmacher Institute. (septiembre-2015). Increase in STI Risk Seen For Women Using Injectable Contraceptives. Recuperado: Abril 25,2018, de Guttmacher Institute. Sitio web: https://www.guttmacher.org/journals/ipsrh /2015/09/increase-sti-risk-seen-womenusing-injectable-contraceptives.

25. Lalinde-Ángel MI, Molina-Marín G, OlarteLezcano ML. Characteristics of users of the birth control program. 2007 National Health survey, Colombia. Invest Educ Enferm. 2013:31(1);20-25.

26. Ley 23 de 18 de febrero de 1981, Presidencia de la República de Colombia, por la cual se dictan normas en materia de ética médica. Recuperado a partir de: https://docs.supersalud.gov.co/PortalWeb/ Juridica/Leyes/L0023_81.pdf.

27. Gaitonde R, Oxman AD, Okebukola PO, Rada G. Interventions to reduce corruption in the health sector (Review). Cochrane library (ON LINE). 2016:8;1-66. DOI: 10.1002/14651858.CD008856.pub2.

28. Pande S, Hiller JE, Nkansah N, Bero L. The effect of pharmacist-provided nondispensing services on patient outcomes, health service utilisation and costs in lowand middle-income countries (Review). Cochrane library (ON LINE). 2013:2;1-60. DOI: $10.1002 / 14651858 . C D 010398$.

29. Thamby S, Subramani P. Seven-Star pharmacist concept by World Health Organization. J Young Pharm. 2014:6(2):1-3. DOI: 10.5530/jyp.2014.2.1.

30. Clavijo G, González V. Caracterización de la dispensación y la administración de un anticonceptivo inyectable frente a la normatividad vigente en Colombia. Rev Invest Innov Salud. 2017: 1;17-30.

31. Proyecto de Ley 246/2016c de 2016, Ponente Padaui-Álvarez $\mathrm{HJ}$, por el cual se dictan medidas para defender el acceso de los usuarios del sistema de salud a medicamentos de calidad, eficacia y seguridad. Recuperado a partir de: http://www.camara.gov.co/medicamentos0 .

32. Asociación de Laboratorios Farmacéuticos de Investigación y Desarrollos. Aportes de la Industria a las asociaciones de pacientes. 2015 [Internet]. Bogotá, D.C. AFRIDRO [citado 27 de abril de 2018]. 1p. Recuperado a partir de: https://www.afidro.org/sites/default/files/A poyos-Asociaciones-de-Pacientes-2.pdf.

33. Corte Constitucional República de Colombia Sentencia C-084/13, Magistrada Ponente Calle-Correa MV, por el cual se declara exequible el artículo 133 de la Ley 1474 de 2011. Recuperado a partir de: http://www.corteconstitucional.gov.co/REL ATORIA/2013/C-084-13.htm.

34. Cámara de la Industria Farmacéutica-ANDI. (2017). Código de Ética y Transparencia. 
Recuperado: Abril 27, 2018, de 2017 Sitio web:http://www.andi.com.co/Uploads/COD IGO\%20DE\%20ETICA_CSD_WEB.pdf.

35. Usma A. La sociedad reclama un mercado farmacéutico más ético [Tesis Especialista en Alta Gerencia][Bogotá, D.C]:Universidad Militar Nueva Granada; 2014[citado 27 de abril de 2018]; Recuperado a partir de: http://repository.unimilitar.edu.co/bitstrea $\mathrm{m} / 10654 / 12142 / 1 /$ La\%20sociedad\%20recl ama\%20un\%20mercado\%20farmac\%C3\% A9utico\%20mas\%20\%C3\%A9tico.pdf. 